\title{
EFFICACY OF HERBAL AND NATURALLY-DERIVED DIETARY SUPPLEMENTS FOR THE MANAGEMENT OF KNEE OSTEOARTHRITIS: A MINI-REVIEW
}

DOI: 10.36740/WLek202108134

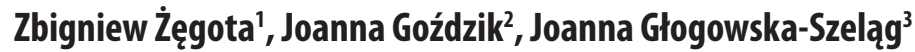 \\ 'ZBIGNIEW ŻĘGOTA SPECJALISTYCZNY OŚRODEK LECZNICZO-BADAWCZY, OSTRODA, POLAND \\ ${ }^{2}$ SILMEDIC LTD, KATOWICE, POLAND \\ ${ }^{3}$ DEPARTMENT OF PATHOPHYSIOLOGY AND ENDOCRINOLOGY, MEDICAL UNIVERSITY OF SILESIA, KATOWICE, POLAND
}

\begin{abstract}
Knee osteoarthritis $(\mathrm{OA})$ accounts for approximately $85 \%$ of the burden of $\mathrm{OA}$ worldwide. Knee $\mathrm{OA}$ is a whole joint disorder involving structural alterations in the hyaline articular cartilage, subchondral bone, ligaments, capsule, synovium, and periarticular muscles. The complex knee OA pathogenesis includes mechanical, inflammatory, and metabolic factors, eventually leading to the synovial joint's structural destruction and failure. This review aims to present an overview of current knowledge on dietary supplements, such as glucosamine, chondroitin, methylsulfonylmethane, diacerein, avocado-soybean unsaponifiables, curcuminoids, as well as boswellic acids. Results originating from several small studies with natural products in managing knee $0 \mathrm{~A}$ are encouraging. However, additional well-designed placebo-controlled clinical trials are required.
\end{abstract}

KEY WORDS: curcuminoids, osteoarthritis, boswellic acids, ginger, complementary and alternative medicines, CAM

Wiad Lek. 2021;74(8):1975-1983

\section{INTRODUCTION}

Osteoarthritis (OA) is a common and disabling disorder representing a substantial and increasing health burden with significant implications for the individuals affected, health-care systems, and wider socioeconomic costs. Considering aging and increasing obesity, and increasing numbers of joint injuries, this already burdensome syndrome is becoming more prevalent, with worldwide estimates indicating that 250 million people are affected [1].

In clinical practice, the knee is the most frequent OA site, followed by the hand and hip. A knee OA stands for $85 \%$ of the OA burden worldwide [2]. The medical cost of OA in high-income countries was estimated to account for $1 \%-2.5 \%$ of the gross domestic product, with hip and knee joint replacements representing a significant proportion of these health-care costs [3]. In the case of knee OA, substantial evidence indicates several moderate to strong risk factors, such as female sex, obesity, and previous knee injury [4]. Knee malalignment is also a moderate to strong risk factor, and knee extensor muscle weakness is likely to be a weak risk factor $[5,6]$.

Currently, treatment of OA comprises lifestyle changes (e.g., weight reduction with exercise and diet), physical aids (e.g., canes or braces), physical therapies, and medications including acetaminophen, non-steroidal anti-inflammatory drugs (NSAIDs), corticosteroids, and/or complementary and alternative medicines (CAMs) [7].

A couple of years ago, Liu et al. prepared a meta-analysis assessing various CAMs [8]. Authors evaluated twenty
CAMs in 69 studies. They demonstrated that Curcuma longa extract, Boswellia serrata extract, collagen hydrolysate, passion fruit peel extract, pycnogenol, and L-carnitine exerted an evident beneficial impact on pain decrease. A smaller impact was observed in the case of avocado soybean unsaponifiables, chondroitin, diacerein, glucosamine, undenatured type II collagen, and methylsulfonylmethane.

\section{THE AIM}

This review aims to present an update on current knowledge of the most commonly used and newly available dietary supplements in knee OA management.

\section{REVIEW AND DISCUSSION}

\section{PATHOPHYSIOLOGY OF OSTEOARTHRITIS}

Osteoarthritis is a whole joint disorder involving structural alterations in the hyaline articular cartilage, subchondral bone, ligaments, capsule, synovium, and periarticular muscles. The complex OA pathogenesis includes mechanical, inflammatory, and metabolic factors, eventually leading to structural destruction and failure of the synovial joint $[9,10]$.

Osteoclasts originate from hematopoietic cells that also give the beginning for macrophages and monocytes. The Receptor Activator NF- $\kappa B$ Ligand (RANKL) is synthe- 
sized by chondrocytes, osteoblasts, and stromal. After RANK activation due to binding to RANKL in the osteoclast precursor cells' membrane, these cells differentiate into osteoclasts [11]. To hamper osteoclasts' activation, osteoprotegerin (OPG), also synthesized in osteoblasts, plays a decoy receptor's role and prevents binding of RANKL to RANK. In consequence, the osteoclastogenesis process is not initiated. The OPG also plays a role in triggering apoptosis of mature osteoclasts. Thus, the RANKL/ OPG ratio is an excellent index to analyze the occurrence of osteogenesis or osteoclastogenesis. When RANKL/ OPG ratio increases, bone destruction dominates, and when this index decreases, subchondral bones are more protected. In OA, various interleukins and cytokines such as IL-1 $\beta$, IL-6, IL-11, IL-17, and TNF- $\alpha$ predispose to increased RANKL formation as well as decreased OPG synthesis, leading to bone loss [12].

Additionally, several other proteases are involved in OA, such as matrix metalloproteinases 3, 9, and 13 (MMP-3, MMP-9, MMP-13), tartrate-resistant acid phosphatase (TRAP), or metalloproteinase with thrombospondin Motifs (ADAMTS). IL-1 acts on chondrocytes, resulting in the induction of NF- $\kappa$ B and activator protein 1 (AP-1) and the production of MMPs, enzymes that breakdown collagen. Among the metalloproteinase, MMP-13 is more potent in the cleavage of type II collagen. The ADAMTS acts in cleaving aggrecan molecules (another component of cartilage). In OA, these proteases are increased, leading to abnormal destruction of cartilage. IL- 6 also plays a significant role in chondrocytes by diminishing type II collagen synthesis. Probably, TNF- $\alpha$ works in synergy with these interleukins in the inhibition of proteoglycan synthesis and increasing cartilage resorption $[13,14]$.

\section{GLUCOSAMINE/CHONDROITIN}

Glucosamine is predominantly found at the level of connective tissue and cartilage. At the articular level, it is an essential precursor for glycosaminoglycan in the production of hyaluronic acid, keratin sulfate, chondroitin sulfate, aggrecan, and collagen type II, which are vital components of the cartilage matrix. Glucosamine inhibits the synthesis of the vital cleavage enzymes in the cartilage, MMP, resulting in a decreased proteoglycan degradation. Additionally, glucosamine hampers proinflammatory processes. Jerosch et al. proved that glucosamine $(1.5 \mathrm{~g}$ per day) significantly decreased the total knee replacement rate in subjects with knee OA (from 14.5 to 6.3\%) and ceased the joint space loss progression [15]. Other studies also confirmed the glucosamine's chondroprotective properties [16]. These properties are believed to be associated with diminished chondrocyte apoptosis, a decrease in MMP-3 levels, and an increase in TGF- 31 and connective tissue growth factor (CTGF) levels [17]. Unfortunately, Kwoch et al. disclosed no structural improvements in MRI knee appearance and CTX-II urinary excretion in subjects using glucosamine in a dose of 1.5 g per day for 24 weeks [18].
Chondroitin sulfate is an essential component of the extracellular matrix, being the most frequent glycosaminoglycan in the aggrecan molecule within the cartilage. Several clinical studies proved the clinically significant structure-modifying impact in subjects treated with chondroitin $(0.8-1.2 \mathrm{~g}$ per day). Two meta-analyses showed the importance of chondroitin in delaying the progression of knee OA $[16,19]$. Chondroitin acts via several mechanisms in the cartilage. It decreases ADAMTS-4, ADAMTS-5, MMP-3, and MMP-13 levels, IL-1ß expression as well as chondrocytes' apoptosis. Chondroitin also increases hyaluronic acid, proteoglycan, and type II collagen synthesis, as well as chondrocytes' metabolism [15]. European League Against Rheumatism (EULAR) granted chondroitin sulfate the highest level of recommendation. Consequently, chondroitin sulfate should be considered a structure-modifying drug in $\mathrm{OA}$, mainly in higher doses (1.2 g per day) [20].

Also, recently Rubbio-Teres showed that due to its better tolerability profile, chondroitin/glucosamine treatment is expected to prevent thousands of adverse events over the next three years and generate considerable savings for the national health system comparing with non-steroidal anti-inflammatory drugs and cyclooxygenase 2 inhibitors [21].

\section{METHYLSULFONYLMETHANE}

Methylsulfonylmethane (MSM) is an organosulfur compound popularly used as an anti-inflammatory agent. Several studies disclosed decreased cartilage degeneration in subjects receiving MSM. This is associated with reduced TNF- $\alpha$ expression, as well as decreased levels of IL-6, NF$\kappa B$, and COX-2 [22]. Previous study with knee OA subjects suggested an improvement in pain and physical function MSM supplementation (3 g twice a day) [23]. However, a recent study by Tennent et al. disclosed that MSM supplementation was not associated with any improvement in the 5 KOOS (Knee Osteoarthritis Outcome Score) subscales or the 6 POMS (Profile of Moods States) subscales at one or two months [24].

\section{DIACEREIN}

Diacerein is an anthraquinone derivate identified in herbal remedies, e.g., yellow dock. It suppresses IL-1ß, MMP-13, and TNF- $\alpha$, also reducing osteoclast formation. Randomized controlled trials showed a positive effect on hip and knee osteoarthritis, but no effects were observed in hand osteoarthritis [25]. Although experts concluded a similar effect of diacerein to NSAIDs; its use is restricted in Europe and the USA due to its adverse reactions (diarrhea, hepatobiliary reactions, and skin reactions). Nevertheless, Pelletier et al. proved in a randomized clinical trial (DISSCO trial) that diacerein was non-inferior to celecoxib in decreasing knee OA pain and improving physical function. Diacerein also characterized a favorable safety profile [26]. 


\section{AVOCADO-SOYBEAN UNSAPONIFIABLES}

Avocado-soybean unsaponifiablea, or piascledine, are a plant-originated extracts comprising of one third avocado oil and two thirds soybean oil. It inhibits IL-1 and also stimulates the synthesis of collagen in cultures. A randomized clinical trial showed that $300 \mathrm{mg}$ of piascledine administration for three years reduced the joint space narrowing changes compared to the control group [27]. Another clinical trial comparing $300 \mathrm{mg}$ daily of piascledine with chondroitin sulfate for six months revealed a similar favorable effect in the case of WOMAC score, represented by a $50 \%$ decrease [28].

Simental-Mendia et al., in their meta-analysis, proved the beneficial effect of avocado-soybean unsaponifiable treatment in symptomatic knee OA but not in hip OA. Additionally, adverse events were comparable in subjects applying avocado-soybean unsaponifiable or placebo [29].

\section{CURCUMIN}

Turmeric (Curcuma longa) belongs to ginger family, which grows commonly in southern and south western regions of tropical. Turmeric has been applied for ages in India and China for treating infection, dermatologic diseases, stress, and depression. Turmeric's impact on human organisms is mainly linked with an orange-yellow, lipophilic polyphenol compound, i.e., curcumin. Curcumin is obtained from the herb's rhizomes [30].

Curcumin hampers RANKL-mediated osteoclastogenesis and TNF- $\alpha$ levels. Bharti et al. proved that RANKL- and curcumin-stimulated macrophages differentiate into osteoclasts to a smaller extent than when only RANKL-stimulated [31]. Additionally, when incubated with curcumin/RANKL liposomes, these cells became smaller and characterized a diminished number of nuclei compared with macrophages incubated only with RANKL. These observations support the impacts of curcuminoids on the process of osteoclastogenesis [32].

Curcumin diminishes the proinflammatory cytokine-induced activation of NF- $\mathrm{kB}$ [31]. Moreover, TRAP and cathepsin $\mathrm{K}$ decreased expression promotes further osteoclastogenesis suppression [32]. Curcuminoids supplementation is associated with an increased OPG/ RANKL, i.e., indicating bone development. In addition to the inhibition of osteoclastogenesis, curcumin may also inhibit pit creation [31].

Apart from postponing bone degradation, curcumin presents chondroprotective effects. It inhibits the production of MMP-1, MMP-3, MMP-9, MMP-13 [32]. Curcumin also restarts the synthesis of type II collagen and glycosaminoglycan and has an anti-apoptotic effect on chondrocytes. Another result is the inhibition of the expression of ADAMT-5. It is a vital property since aggrecanase-mediated aggrecan degradation occurs in early-stage OA [12].

Curcumin also exerts anti-inflammatory properties. In a number of studies, it was proved that curcumin might down-regulate phospholipase A2, cyclooxygenase-2, and lipoxygenase expression as well as decrease levels of IL- $1 ß$, IL-6, IL-8, PGEs, and TNF- $\alpha$. It also acts as an inducer of apoptosis in synoviocytes, decreasing the inflammation process [33].

In 2014 a randomized, double-blind, placebo-controlled trial from Iran showed curcuminoid-receiving subjects characterized significantly lower scores on the Western Ontario and McMaster Universities Osteoarthritis Index (WOMAC) and Lequesne's pain functional index than subjects receiving placebo [34]. However, earlier two studies by Belcaro et al. proved the clinical efficacy and safety of Meriva, a phosphatidylcholine complex with curcumin designed to enhance its oral bioavailability [35]. The first study, a short-term product registry study, showed that the group receiving Meriva had improvements in pain sensation, joint stiffness, and physical function according to their WOMAC scores [36]. The second study aimed to assess the long-term efficacy and safety of Meriva in subjects with OA [37]. In this study, subjects in both the control group, which was defined as the "best available treatment," and the treatment group, which was the "best available treatment plus Meriva," could use NSAIDs during the study to control their pain if needed. Subjects from the treatment group had improvements in pain, stiffness, and physical function according to their WOMAC scores, improved Karnofsky performance scores, and statistically significant reductions in serum concentrations of SCD40L, IL-1 $\beta$, IL-6, and ESR. Additionally, the treatment group showed a $63.4 \%$ decrease in NSAIDs use compared with $8 \%$ in the control group and a $63.5 \%$ decrease in health care costs compared with $3.7 \%$ in the control group. This study suggested that curcumin might be used instead of NSAIDs. This option would be especially attractive for subjects who cannot tolerate NSAIDs, have a history of adverse gastrointestinal and renal effects, or simply wish to take more natural substances.

Moreover, a proprietary lecithin formulation of curcumin (Meriva) was shown to reduce acute pain subjects with various chronic states [38]. Meriva supplementation $(2.0 \mathrm{~g})$ exerted proven analgesic activity comparable with acetaminophen $(1.0 \mathrm{~g})$ but lower than nimesulide (100 $\mathrm{mg}$ ). Worth stressing is that gastrointestinal tolerance was much better than in the case of nimesulide and comparable with acetaminophen. Although this Meriva dose was definitely larger than that administered in persistent inflammatory disorders (1.0 - $1.2 \mathrm{~g}$ per day), Meriva pain-relieving properties could be associated with anti-inflammatory effects induced by curcumin in such an increased dose.

Another interesting aspect is the delayed onset muscle soreness (DOMS) evoked by eccentric muscle activity. DOMS is linked with the inflammatory response and reactive oxygen species synthesis. Drobnic et al. assessed in a randomized, placebo-controlled trial the hypothesis whether curcumin had the potential to diminish tissue injury due to inflammation and oxidative stress associated with the eccentric continuous exercise [39]. 
Twenty male moderately active healthy volunteers were enrolled to curcumin (200 mg twice per day) or placebo. Curcumin administration was launched two days prior to the downhill running test and maintained 24 hours after the test (in total -4 days). Subjects taking curcumin reported less lower limb pain than placebo group subjects. Moreover, significantly fewer subjects in the curcumin group revealed evidence of muscle damage in MRI. This study suggested that curcumin might bear a potential role in preventing DOMS, as indicated by its impact on pain intensity and muscle injury.

Di Pierro et al. observed similar findings in rugby players [40]. Fifty rugby players with musculoskeletal pain in the course of traumatic injuries, physical overload, or acute episodes of chronic pain were enrolled. They received standard analgesic drugs $(n=25)$ or Meriva-based supplement $(\mathrm{n}=25)$ for maximally 10 days. The pain perception and the functions were evaluated at baseline and after 1, 3, 6, 10, and 20 days from the treatment initiation. Authors concluded that curcumin-based supplements could stand as a safe and promising alternative in painful musculoskeletal disorders in subjects exposed to intense physical activities.

Belcaro et al. also confirmed that the 4-month supplementation of glucosamine together with Meriva could promote the faster onset of action and better outcomes than using the combination of glucosamine and chondroitin sulfate in subjects with OA [41].

Moreover, Franceschi et al. evaluated the impact of Meriva supplementation in otherwise healthy elderly subjects [42]. Several parameters (handgrip, weight lifting, time/distance before feeling tired after cycling, walking, climbing stairs; general fitness, proteinuria, oxidative stress, Karnofsky scale; left ventricular ejection fraction) were assessed at baseline and after three months. Authors proved that Meriva's addition to a standardized diet and exercise plan was associated with increased strength and physical performance in elderly subjects (sarcopenia prevention). Moreover, Riva et al. suggested that a curcumin-based supplementation combined with an appropriate lifestyle could prevent osteopenia in many cases [43].

To sum up, one should mention a systematic review by Onakpoya et al. The authors investigated the efficacy of curcuminoids administered orally in OA [44]. The authors included seven studies with a total number of 797 patients with primary knee OA. Curcuminoids administration, compared with placebo, significantly diminished knee pain $(\mathrm{p}<0.01)$ and improved quality of life $(p<0.01)$. Curcuminoid use was also associated with significant improvements in WOMAC total scores as well as with substantial reductions in the use of rescue medication. The summary of curcumin properties is presented in Figure 1.

\section{BOSWELLIC ACIDS}

Boswellia serrata is a tree encountered in mountainous areas of India, Middle East and Northern Africa. As stated by Siddiqui "oleo gum-resin is tapped from the incision made on the trunk of the tree and is then stored in specially made bamboo basket for removal of oil content and getting the resin solidified. After processing, the gum-resin is then graded according to its flavor, color, shape and size. Gum-resin extracts of Boswellia serrata have been traditionally used in folk medicine for centuries to treat various chronic inflammatory diseases. The resinous part of Boswellia serrata possesses monoterpenes, diterpenes, triterpenes, tetracyclic triterpenic acids and four major pentacyclic triterpenic acids, responsible for anti-inflammatory properties" [45]. Recently researchers have verified the efficacy and safety of Boswellin, a novel extract of Boswellia serrata extract (BSE) containing 3-acetyl-11-keto- $\beta$-boswellic acid (AKBBA) with $\beta$-boswellic acid (BBA) in a double-blinded, placebo-controlled trial [46]. The results proved that $\mathrm{AKBBA}$ and $\mathrm{BBA}$ exerted synergistic anti-inflammatory/anti-arthritic effects on physical and functional ability and decreased pain and stiffness.

\section{GINGER}

Ginger (Zingiber officinale) is a traditional herb. Ginger is applied not only as a spice, but also used as a remedy to manage different ailments, e.g., musculoskeletal disorders, migraine, and even diabetes. There are two essential components, 6-gingerol and 6-shogaol, in ginger. They are responsible for preventing inflammation and oxidative stress [47]. In a randomized trial in elderly subjects with knee OA, authors disclosed that ginger supplementation was linked with decreased levels of TNF- $\alpha$ and IL-1 $\beta$ [48]. More recently, meta-analysis results showed that oral, but not topical, ginger was associated with pain relief in knee OA [49].

\section{NOVEL HERBAL COMBINATIONS}

\section{CURCUMIN WITH BOSWELLIC ACIDS}

Haroyan et al. proved that subjects taking curcuminoids combined with boswellic acids performed better than subjects taking placebo in physical performance tests and the WOMAC joint pain index. In contrast, when only the curcuminoid complex extract was used, it was more effective than placebo only in physical performance tests [50]. A further meta-analysis by Bannuru et al. confirmed that curcuminoids and Boswellia formulations might pose a valuable additive to the knee OA treatment regimens [51]. They effectively relieve pain symptoms and simultaneously reduce safety risks.

\section{GARLIC, CELERY, AND DEVIL'S CLAW}

Garlic (Allium sativum) use was registered, among others, over 5000 years ago in Ayurvedic medicine, and over 3000 years ago in ancient China. The active substances enclosed in garlic include sulfur-containing elements such as alliin, substances derived from alliin (e.g., allicin) or enzymes (e.g., alliinase). Allicin is the key bioactive substance in 


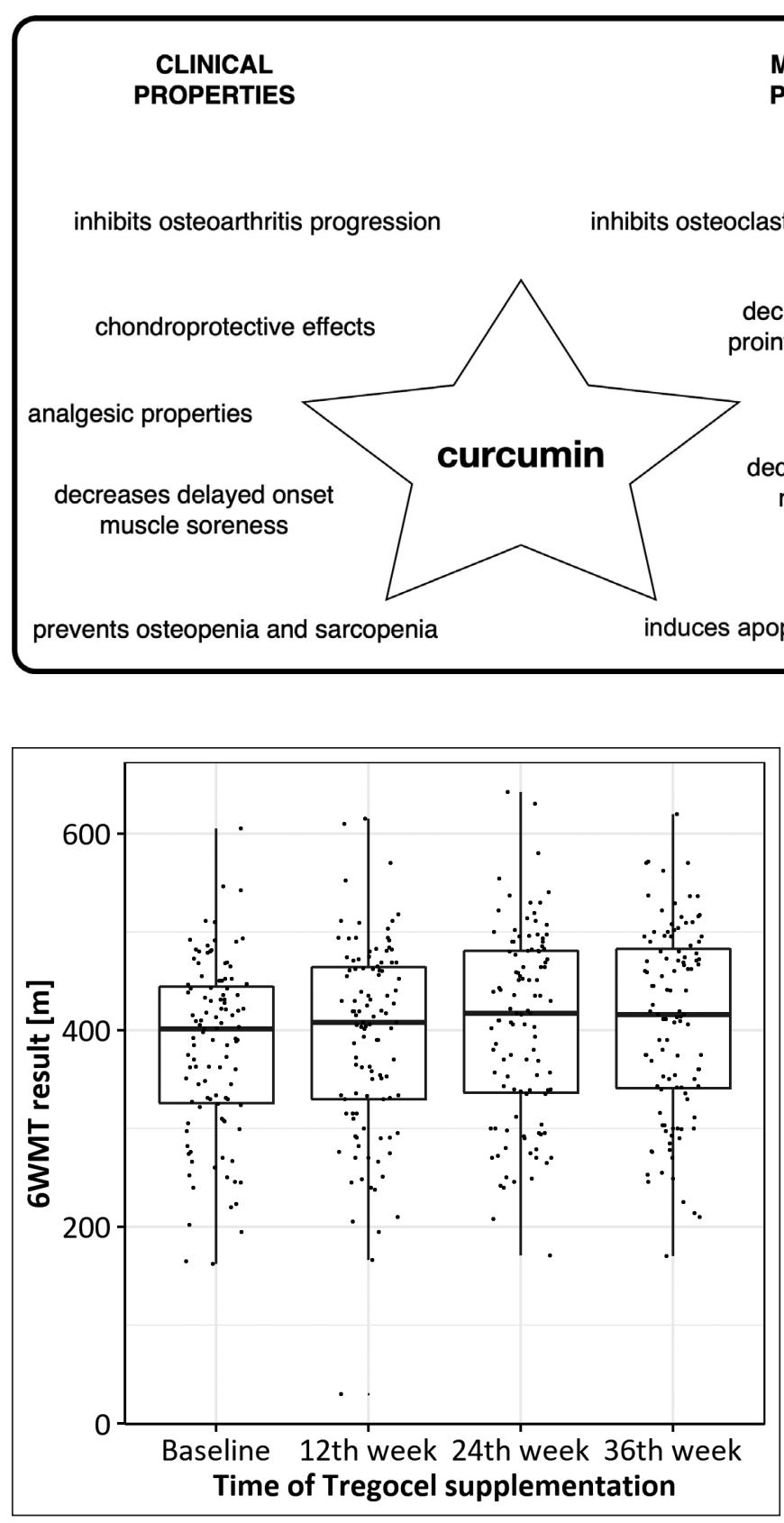

Fig. 2. Results of 6-minute walk test (6MWT) at visit 1, visit 5 (after 12 weeks), visit 9 (after 24 weeks), and visit 13 (after 36 weeks). Results are presented as median with interquartile range (IQR).

the garlic extract as well as raw garlic derivatives [52]. Dehghani et al. evaluated the anti-inflammatory and analgesic properties of garlic supplementation on pain severity and resistin and TNF- $\alpha$ serum levels in women with overweight or obesity and knee OA [53]. After 12 weeks, resistin levels were significantly reduced in the garlic group $(6.41 \pm 2.40$ to $5.56 \pm 2.16 \mathrm{ng} / \mathrm{mL} ; \mathrm{p}<0.01)$. Similarly, pain scores were markedly decreased in the garlic group $(6.8 \pm 2$ to $5.3 \pm 2.3 ; \mathrm{p}<0.01)$, but not in the placebo group $(6.7 \pm 2.4$ to $6.2 \pm 2.5 ; \mathrm{p}=0.67)$. At the end of the study, the difference in pain scores was also statistically significant between groups $(5.3 \pm 2.3$ vs.
MOLECULAR PROPERTIES

Fig. 1. Properties of curcumin.

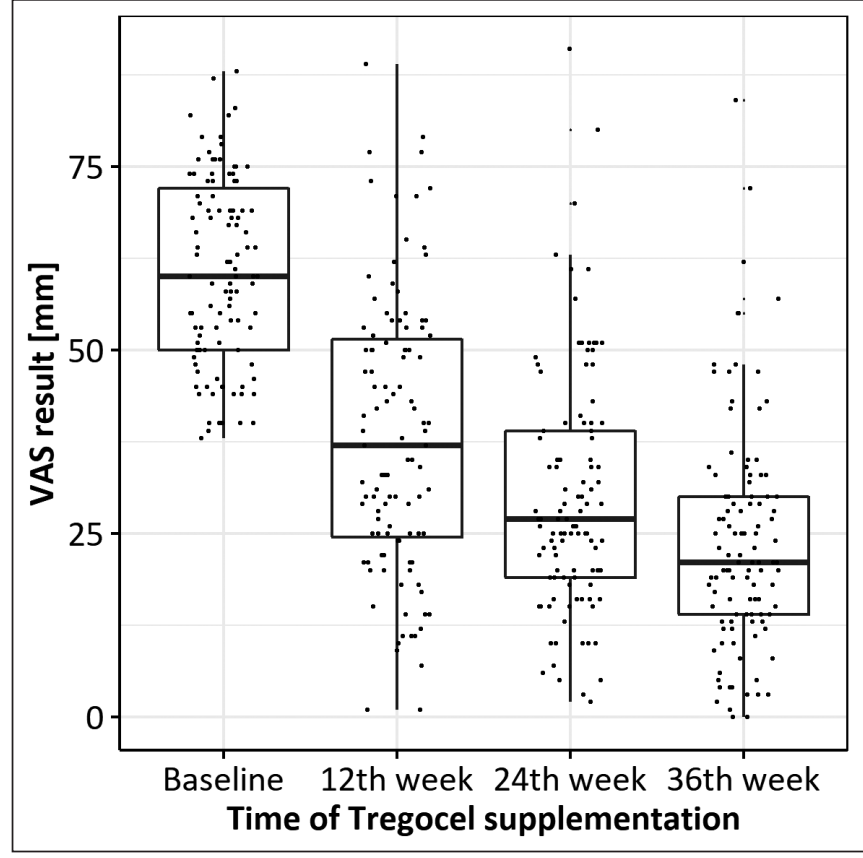

Fig. 3. Pain assessment in VAS scale at visit 1, visit 5 (after 12 weeks), visit 9 (after 24 weeks), and visit 13 (after 36 weeks). Results are presented as median with interquartile range (IQR).

$6.2 \pm 2.5 ; \mathrm{p}=0.04)$. The results confirmed that 3 -month garlic supplementation might diminish pain severity in overweight or obese women with knee OA. Hedaya R proved the benefits of using celery's extracts, and Denner SS - devil's claw in OA $[54,55]$.

The main compounds of devil's claw are iridoid glycosides, such as harpagoside, harpagide, and procumbide. Interestingly, the devil's claw is used orally in folk medicine to treat a wide range of health conditions, including indigestion, fever, allergic reactions, and rheumatism. Additionally, other ethnomedicinal applications include urinary infectious, postpartum pain, ulcers, and inflam- 
matory bowel diseases. Recently, it was shown that devil's claw was beneficial in rheumatoid arthritis (significant reduction in global pain and stiffness alongside with significant improvements in function and mean pain scores for hand, wrist, elbow, shoulder, hip, knee and back pain) as well as in osteoporosis (bone mineral density stimulation) [56].

\section{CURCUMINOIDS, BOSWELLIC ACIDS, CELERY SEED, DEVIL'S CLAW AND GINGER}

An example of a novel supplement containing clinical levels of these key medicinal herbs is Tregocel', which listed as a complementary medicine in Australia, and manufactured according to pharmaceutical GMP standards. The product is an herbal composite formulation that is designed to provide supportive benefits to standard pharmacotherapies for OA. It contains a patented curcuminoid preparation and standardized extracts of the herbs Harpagophytum procumbens, Boswellia serrata, Apium graveolens, and Zingiber officinale. Curcumin is an active phenolic compound found in turmeric (Curcuma longa). In Tregocel, curcumin is incorporated into a phospholipid complex (Meriva, Trademark of Indena, S.p.A.), which was shown previously to be 29 -fold more absorbed than natural, unformulated curcuminoids [36]. It also contains a standardized extract of Harpagophytum procumbens, with several active compounds, including harpagosides as the primary compounds. Tregocel also includes an extract of Boswellia serrata, in which boswellic acids are the primary active constituents. Apium graveolens (celery) seeds are other components, which contain volatile oils such as limonene and selinene, flavonoid compounds, celeroside glucosides, and phthalide glycosides, as well as aromatic and lignin glucosides. Finally, Tregocel ${ }^{\circ}$ contains Zingiber officinale (ginger) extract, with the main phytochemicals as phenols (gingerol, shogaols, paradols), diarylheptanoids, gingerdiols, and sesquiterpenes [57].

An exploratory study with Tregocel ${ }^{\circ}$ was performed in 2019 and assessed physical performance with a 6-minute walk test (6MWT) and WOMAC indeces in participants with symptomatic mild knee OA (up to grade 2), as well as the perception of pain, general performance, level of standard pharmacological treatment and basic safety parameters, performed according to a registered clinical trial protocol[58]. One hundred and seven subjects completed a 36-week intervention across eight clinical sites in Poland. In brief, the intervention was associated with increased physical capacity and decreased pain. At baseline, the mean 6MWT distance was $382.8 \pm 88.1 \mathrm{~m}$, and at the end of the study this had extended to 408.8 $\pm 96.3 \mathrm{~m}$. The increase of $26.0 \pm 30.4 \mathrm{~m}$ was statistically significant $(\mathrm{p}<0.001)$ (Figure 2$)$. WOMAC scores assessed before the 6MWT in all domains (pain, stiffness, physical function, and total) had improved progressively throughout the study. The subsequent visits' scores were statistically significantly lower than initial values $(\mathrm{p}<$
0.001 for all comparisons). The decrease was the greatest after 12 weeks of Tregocel" supplementation (about 50\% in all domains) and continued to improve in subsequent visits. With the duration of the study, subjective pain levels significantly decreased from baseline [60.0 (IQR 50.0 - 72.0) at baseline] to 37.0 (IQR 24.5 - 51.5) after 12 weeks of supplementation ( $<<0.001$ ), 27.0 (IQR $19.0-$ 39.0) after 24 weeks $(\mathrm{p}<0.001)$ and 21.0 (IQR 14.0 $30.0)$ after 36 weeks $(\mathrm{p}<0.001)$. The overall decrease was statistically significant, and the value at the end of the study stood at approximately $30 \%$ of baseline pain scores (Figure 3). Concomitantly, a decrease in the requirement for standard medications was also observed. At baseline, $99.1 \%$ of patients regularly took anti-inflammatory/analgesic drugs, whereas this progressively declined after 12 weeks (76.6\%), 24 weeks (69.2\%), and 36 weeks (55.1\%). As with previous trials of polyherbal supplements, the outcomes of this intervention are clinically meaningful for OA treatment, and integrate easily with standard therapies.

\section{CLINICAL IMPLICATIONS}

Nutraceuticals in terms of being promising anti-inflammatory and antioxidant agents undergo extensive research studies. A growing body of evidence has suggested that many plants display robust pharmacological properties (anti-inflammatory, anti-catabolic, and anti-apoptotic), and that many of them are able of protecting joint cartilage against destructive processes. Furthermore, several studies have proved that the earlier mentioned pro-inflammatory cytokines are mostly regulated by the key transcription factor, i.e., NF- $\kappa \mathrm{B}$.

Analyzing the position of nutraceuticals in the management of subjects with knee OA, one must be stressed that we require new strategies and large-scale clinical trials that are supposed to take several years. Although we can identify some research revealing merit as well as nutraceuticals' efficacy comparable with NSAIDs, frequently these studies characterize small population, short follow-up or the lack of control group.

Nevertheless, nutraceuticals can become a very useful addition to pain relievers (e.g., NSAIDs), which are linked with various side effects and are used commonly in large quantities worldwide. Nutraceuticals might have their place especially in subjects with mild or moderate OA stages.

\section{CONCLUSIONS}

Knee OA, the most frequent joint disorder, is linked with substantial health-care expenses, impaired productivity, as well as diminished quality of life. However, available pharmaceutical treatments have limitations in terms of efficacy and long-term safety. Results originating from several small studies with natural products in managing knee OA are encouraging and may allow for greater flexibility in clinical settings. 


\section{REFERENCES}

1. Prieto-Alhambra D, Judge A, Javaid MK, et al. Incidence and risk factors for clinically diagnosed knee, hip and hand osteoarthritis: influences of age, gender and osteoarthritis affecting other joints. Ann Rheum Dis. 2014; 73(9): 1659-1664, doi: 10.1136/annrheumdis-2013-203355

2. Safiri S, Kolahi AA, Smith E, et al. Global, regional and national burden of osteoarthritis 1990-2017: a systematic analysis of the Global Burden of Disease Study 2017. Ann Rheum Dis. 2020;79(6):819-828, doi: 10.1136/ annrheumdis-2019-216515

3. Hunter DJ, Bierma-Zeinstra S. Osteoarthritis. Lancet. 2019;393(10182):1745-1759, doi: 10.1016/S0140-6736(19)30417-9

4. Silverwood V, Blagojevic-Bucknall M, Jinks C, et al. Current evidence on risk factors for knee osteoarthritis in older adults: a systematic review and meta-analysis. Osteoarthritis Cartilage. 2015;23(4):507-515, doi: 10.1016/j.joca.2014.11.019

5. Runhaar J, van Middelkoop M, Reijman M, et al. Malalignment: a possible target for prevention of incident knee osteoarthritis in overweight and obese women. Rheumatology (0xford). 2014;53(9):1618-1624, doi: 10.1093/rheumatology/keu141

6. Oiestad BE, Juhl CB, Eitzen I, et al. Knee extensor muscle weakness is a risk factor for development of knee osteoarthritis. A systematic review and meta-analysis. Osteoarthritis Cartilage. 2015;23(2):171-177, doi: 10.1016/j.joca.2014.10.008

7. Sokolove J, Lepus CM. Role of inflammation in the pathogenesis of osteoarthritis: latest findings and interpretations. Ther Adv Musculoskelet Dis. 2013;5(2):77-94, doi: 10.1177/1759720X12467868

8. Liu X, Machado GC, Eyles JP, et al. Dietary supplements for treating osteoarthritis: a systematic review and meta-analysis. Br J Sports Med. 2018; 52(3):167-175, doi: 10.1136/bjsports-2016-097333

9. Martel-Pelletier J, Barr AJ, Cicuttini FM, et al. Osteoarthritis. Nat Rev Dis Primers. 2016;2:16072, doi: 10.1038/nrdp.2016.72

10. LoeserRF,Collins JA, Diekman BO. Ageingand the pathogenesis of osteoarthritis. Nat Rev Rheumatol. 2016;12(7):412-420, doi: 10.1038/nrrheum.2016.65

11. Tamma R, Zallone A. Osteoblast and osteoclast crosstalks: from OAF to Ephrin. Inflamm Allergy Drug Targets. 2012;11(3):196-200, doi: $10.2174 / 187152812800392670$

12. Akuri MC, Barbalho SM, Val RM, et al. Reflections about Osteoarthritis and Curcuma longa. Pharmacogn Rev. 2017;11(21):8-12, doi: 10.4103/ phrev.phrev_54_16

13. Wojdasiewicz P, Poniatowski LA, Szukiewicz D. The role of inflammatory and anti-inflammatory cytokines in the pathogenesis of osteoarthritis. Mediators Inflamm. 2014; 2014(561459, doi: 10.1155/2014/561459

14. Zhang Z, Leong DJ, Xu L, et al. Curcumin slows osteoarthritis progression and relieves osteoarthritis-associated pain symptoms in a post-traumatic osteoarthritis mouse model. Arthritis Res Ther. 2016;18(1):128, doi: 10.1186/s13075-016-1025-y

15. Jerosch J. Effects of Glucosamine and Chondroitin Sulfate on Cartilage Metabolism in OA: Outlook on Other Nutrient Partners Especially Omega-3 Fatty Acids. Int JRheumatol. 2011;2011:969012, doi: 10.1155/2011/969012

16. Gallagher B, Tjoumakaris FP, Harwood MI, et al. Chondroprotection and the prevention of osteoarthritis progression of the knee: a systematic review of treatment agents. Am J Sports Med. 2015;43(3):734-744, doi: 10.1177/0363546514533777

17. Ali $A A$, Lewis $S M$, Badgley $\mathrm{HL}$, et al. Oral glucosamine increases expression of transforming growth factor beta1 (TGFbeta1) and connective tissue growth factor (CTGF) mRNA in rat cartilage and kidney: implications for human efficacy and toxicity. Arch Biochem Biophys. 2011;510(1):11-18, doi: 10.1016/j.abb.2011.03.014
18. Kwoh CK, Roemer FW, Hannon MJ, et al. Effect of oral glucosamine on joint structure in individuals with chronic knee pain: a randomized, placebo-controlled clinical trial. Arthritis Rheumatol. 2014;66(4):930939, doi: 10.1002/art.38314

19. Knapik JJ, Pope R, Hoedebecke SS, et al. Effects of Oral Chondroitin Sulfate on Osteoarthritis-Related Pain and Joint Structural Changes: Systematic Review and Meta-Analysis. J Spec Oper Med. 19(1):113-124.

20. Arden NK, Perry TA, Bannuru RR, et al. Non-surgical management of knee osteoarthritis: comparison of ESCEO and OARSI 2019 guidelines. Nat Rev Rheumatol. 2020; doi: 10.1038/s41584-020-00523-9

21. Rubio-Terres C. Analysis of the Health and Budgetary Impact of Chondroitin Sulfate Prescription in the Treatment of Knee Osteoarthritis Compared to NSAIDs and COXIBs. Clinicoecon Outcomes Res. 2020;14(12):505-514.

22. Butawan M, Benjamin RL, Bloomer RJ. Methylsulfonylmethane: Applications and Safety of a Novel Dietary Supplement. Nutrients. 2017;9(3), doi: 10.3390/nu9030290

23. Kim LS, Axelrod LJ, Howard P, et al. Efficacy of methylsulfonylmethane (MSM) in osteoarthritis pain of the knee: a pilot clinical trial. Osteoarthritis Cartilage. 2006;14(3):286-294, doi: 10.1016/j.joca.2005.10.003

24. Tennent DJ, Hylden CM, Kocher BK, et al. A randomized controlled trial evaluating methylsulfonylmethane versus placebo to prevent knee pain in military initial entry trainees. US Army Med Dep J. 2017;3-17):21-25.

25. Zhu Z, Li J, Ruan G, et al. Investigational drugs for the treatment of osteoarthritis, an update on recent developments. Expert Opin Investig Drugs. 2018;27(11):881-900, doi: 10.1080/13543784.2018.1539075

26. Pelletier JP, Raynauld JP, Dorais M, et al. An international, multicentre, double-blind, randomized study (DISSCO): effect of diacerein vs celecoxib on symptoms in knee osteoarthritis. Rheumatology (0xford). 2020; doi: 10.1093/rheumatology/keaa072

27. Maheu E, Cadet C, Marty M, et al. Randomised, controlled trial of avocado-soybean unsaponifiable (Piascledine) effect on structure modification in hip osteoarthritis: the ERADIAS study. Ann Rheum Dis. 2014;73(2):376-384, doi: 10.1136/annrheumdis-2012-202485

28. Pavelka K, Coste P, Geher P, et al. Efficacy and safety of piascledine 300 versus chondroitin sulfate in a 6 months treatment plus 2 months observation in patients with osteoarthritis of the knee. Clin Rheumatol. 2010;29(6):659-670, doi: 10.1007/s10067-010-1384-8

29. Simental-Mendia M, Sanchez-Garcia A, Acosta-Olivo CA, et al. Efficacy and safety of avocado-soybean unsaponifiables for the treatment of hip and knee osteoarthritis: A systematic review and meta-analysis of randomized placebo-controlled trials. Int J Rheum Dis. 2019;22(9):16071615, doi: 10.1111/1756-185X.13658

30. Kocaadam B, Sanlier N. Curcumin, an active component of turmeric (Curcuma longa), and its effects on health. Crit Rev Food Sci Nutr. 2017;57(13): 2889-2895, doi: 10.1080/10408398.2015.1077195

31. Bharti AC, Takada Y, Aggarwal BB. Curcumin (diferuloylmethane) inhibits receptor activator of NF-kappa B ligand-induced NF-kappa B activation in osteoclast precursors and suppresses osteoclastogenesis. J Immunol. 2004; 172(10):5940-5947, doi: 10.4049/jimmunol.172.10.5940

32. Yeh CC, Su YH, Lin YJ, et al. Evaluation of the protective effects of curcuminoid (curcumin and bisdemethoxycurcumin)-loaded liposomes against bone turnover in a cell-based model of osteoarthritis. Drug Des Devel Ther. 2015;9:2285-2300, doi: 10.2147/DDDT.S78277

33. Kuptniratsaikul V, Dajpratham P, Taechaarpornkul W, et al. Efficacy and safety of Curcuma domestica extracts compared with ibuprofen in patients with knee osteoarthritis: a multicenter study. Clin Interv Aging. 2014;9:451-458, doi: 10.2147/CIA.S58535 
34. Panahi Y, Rahimnia AR, Sharafi M, et al. Curcuminoid treatment for knee osteoarthritis: a randomized double-blind placebo-controlled trial. Phytother Res. 2014;28(11):1625-1631, doi: 10.1002/ptr.5174

35. Anand $P$, Kunnumakkara $A B$, Newman RA, et al. Bioavailability of curcumin: problems and promises. Mol Pharm. 2007;4(6):807-818, doi: 10.1021/mp700113r

36. Belcaro $G$, Cesarone MR, Dugall $M$, et al. Product-evaluation registry of Meriva(R), a curcumin-phosphatidylcholine complex, for the complementary management of osteoarthritis. Panminerva Med. 2010;52(2 Suppl 1):55-62.

37. Belcaro G, Cesarone MR, Dugall M, et al. Efficacy and safety of Meriva $(R)$, a curcumin-phosphatidylcholine complex, during extended administration in osteoarthritis patients. Altern Med Rev. 2010;15(4):337-344.

38. Di Pierro F, Rapacioli G, Di Maio EA, et al. Comparative evaluation of the pain-relieving properties of a lecithinized formulation of curcumin (Meriva((R))), nimesulide, and acetaminophen. J Pain Res. 2013;6(201205, doi: $10.2147 /$ JPR.S42184

39. Drobnic F, Riera J, Appendino G, et al. Reduction of delayed onset muscle soreness by a novel curcumin delivery system (Meriva(R)): a randomised, placebo-controlled trial. J Int Soc Sports Nutr. 2014; 11(31, doi: 10.1186/1550-2783-11-31

40. Di Pierro F, Zacconi P, Bertuccioli A, et al. A naturally-inspired, curcumin-based lecithin formulation (Meriva(R) formulated as the finished product Algocur(R)) alleviates the osteo-muscular pain conditions in rugby players. Eur Rev Med Pharmacol Sci. 2017;21(21):4935-4940.

41. Belcaro $G$, Dugall $M$, Luzzi $R$, et al. Meriva(R)+Glucosamine versus Condroitin+Glucosamine in patients with knee osteoarthritis: an observational study. Eur Rev Med Pharmacol Sci. 2014;18(24):3959-3963.

42. Franceschi F, Feregalli B, Togni S, et al. A novel phospholipid delivery system of curcumin (Meriva(R)) preserves muscular mass in healthy aging subjects. Eur Rev Med Pharmacol Sci. 2016;20(4):762-766.

43. Riva A, Togni S, Giacomelli L, et al. Effects of a curcumin-based supplementation in asymptomatic subjects with low bone density: a preliminary 24-week supplement study. Eur Rev Med Pharmacol Sci. 2017; 21(7):1684-1689.

44. Onakpoya IJ, Spencer EA, Perera R, et al. Effectiveness of curcuminoids in the treatment of knee osteoarthritis: a systematic review and metaanalysis of randomized clinical trials. Int J Rheum Dis. 2017;20(4):420433, doi: 10.1111/1756-185X.13069

45. Siddiqui MZ. Boswellia serrata, a potential antiinflammatory agent: an overview. Indian J Pharm Sci. 2011;73(3):255-261, doi: 10.4103/0250474X.93507

46. Majeed M, Majeed S, Narayanan NK, et al. A pilot, randomized, doubleblind, placebo-controlled trial to assess the safety and efficacy of a novel Boswellia serrata extract in the management of osteoarthritis of the knee. Phytother Res. 2019;33(5):1457-1468, doi: 10.1002/ ptr.6338

47. Mohd Sahardi NFN, Makpol S. Ginger (Zingiber officinale Roscoe) in the Prevention of Ageing and Degenerative Diseases: Review of Current Evidence. Evid Based Complement Alternat Med. 2019;2019:5054395, doi: 10.1155/2019/5054395

48. Mozaffari-Khosravi H, Naderi Z, Dehghan A, et al. Effect of Ginger Supplementation on Proinflammatory Cytokines in Older Patients with Osteoarthritis: Outcomes of a Randomized Controlled Clinical Trial. J Nutr Gerontol Geriatr. 2016;35(3):209-218, doi: 10.1080/21551197.2016.1206762
49. Araya-Quintanilla F, Gutierrez-Espinoza H, Munoz-Yanez MJ, et al. Effectiveness of Ginger on Pain and Function in Knee Osteoarthritis: A PRISMA Systematic Review and Meta-Analysis. Pain Physician. 2020;23(2):E151-E161.

50. Haroyan A, Mukuchyan V, Mkrtchyan N, et al. Efficacy and safety of curcumin and its combination with boswellic acid in osteoarthritis: a comparative, randomized, double-blind, placebo-controlled study. BMC Complement Altern Med. 2018;18(1):7, doi: 10.1186/s12906017-2062-z

51. Bannuru RR, Osani MC, Al-Eid F, et al. Efficacy of curcumin and Boswellia for knee osteoarthritis: Systematic review and metaanalysis. Semin Arthritis Rheum. 2018;48(3):416-429, doi: 10.1016/j. semarthrit.2018.03.001

52. Zhu Y, Anand $R$, Geng $X$, et al. A mini review: garlic extract and vascular diseases. Neurol Res. 2018;40(6):421-425, doi: 10.1080/01616412.2018.1451269

53. Dehghani S, Alipoor E, Salimzadeh A, et al. The effect of a garlic supplement on the pro-inflammatory adipocytokines, resistin and tumor necrosis factor-alpha, and on pain severity, in overweight or obese women with knee osteoarthritis. Phytomedicine. 2018;48:70-75, doi: 10.1016/j.phymed.2018.04.060

54. Hedaya R. Five Herbs Plus Thiamine Reduce Pain and Improve Functional Mobility in Patients With Pain: A Pilot Study. Altern Ther Health Med. 2017;23(1):14-19.

55. Denner SS. A review of the efficacy and safety of devil's claw for pain associated with degenerative musculoskeletal diseases, rheumatoid, and osteoarthritis. Holist Nurs Pract. 2007;21(4):203-207, doi: 10.1097/01.HNP.0000280932.65581.72

56. Menghini L, Recinella L, Leone $S$, et al. Devil's claw (Harpagophytum procumbens) and chronic inflammatory diseases: A concise overview on preclinical and clinical data. Phytother Res. 2019;33(9):2152-2162, doi: 10.1002/ptr.6395

57. Al-Nahain A, Jahan R, Rahmatullah M. Zingiber officinale: A Potential Plant against Rheumatoid Arthritis. Arthritis. 2014;2014:159089, doi: $10.1155 / 2014 / 159089$

58. Żęgota Z, Goździk J, Głogowska-Szeląg J. Prospective, Multicenter Evaluation of a Polyherbal Supplement alongside Standard-of-Care Treatment for Mild Knee Osteoarthritis. Adv Orthop. 2021 May 7;2021:5589597. doi: 10.1155/2021/5589597

\section{ORCID and contributionship:}

Zbigniew Żęota: 0000-0003-4471-0277 B,E-F

Joanna Goździk: 0000-0002-6435-4794 B,E-F

Joanna Głogowska-Szelag: 0000-0003-0949-322X B,E-F

\section{Acknowledgements:}

The authors thank Jacek Bil, $M D, P h D$, for providing medical writing support. This study was funded by Max Biocare, South Yarra, Australia, in accordance with Good Publication Practice (GPP3) guidelines (http://www.ismpp. org/gpp3).

\section{Conflict of interest:}

The study wasfunded by Max Biocare Pty. Ltd, Melbourne, Australia. Zbigniew Żegota, Joanna Goździk, Joanna Głogowska-Szeląg participated in Tregocel $^{\circ}$ medical experiment sponsored by MaxBiocare and received fees from ClinMed Pharmal Clinscience (MaxBiocare). 


\section{CORRESPONDING AUTHOR}

Joanna Głogowska-Szeląg

Department of Pathophysiology and Endocrinology,

Medical University of Silesia, Poland

e-mail: szelag@poczta.onet.pl

Received: 07.05.2021

Accepted: 30.07 .2021

A - Work concept and design, B - Data collection and analysis, C - Responsibility for statistical analysis,

D-Writing the article, $\mathbf{E}$-Critical review, $\mathbf{F}$ - Final approval of the article 